\title{
Novel Highly Functionalized Benzoylaminocarbothioyl Pyrrolidine from Benzoylisothiocyanate and Substitueted Pyrrolidine Derived From $\alpha$-Aminoasit Ester via Imine -Azomethine Ylide- 1,3-Dipolar Cycloaddition Cascade
}

\author{
H. Ali Dondas ${ }^{*}$ and Ozgul Altınbas ${ }^{b}$ \\ c. Mersin University, Faculty of Pharmacy. Chemistry Department, Yenisehir Campus, 33342, Mersin-Turkey \\ d. Mersin University, Faculty of Science and Arts, Chemistry Department, Mezith, 33342 Mersin-Turkey
}

\begin{abstract}
A series of novel highly functionalized benzoylaminocarbothioyl pyrrolidines were prepared by the reaction of benzoylisothiocyanate with substitueted pyrrolidine derived from $\alpha$-aminoasit ester via metal catalsed imine -azomethine ylides-1,3-Dipolar Cycloaddition in excellent yield. An example of chiral version benzoylaminocarbothioyl pyrrolidine were also reported. The chirality oryginated from menthyl acrylate as chiral dipolarophile in the cycloaddition cascade and induced three novell chiral centres.
\end{abstract}

\section{Introduction:}

There have been many reports in the literature of thiourea moiety containing N,N'-aryl and alkyl ligands and their metal complexes ${ }^{1-2}$. Some of these compounds show a wide range of pharmaceutical activities such as antifungal ${ }^{3.4}$, antitumour ${ }^{5}$ antifoulingl ${ }^{6}$ properties, and screened for the some other biological actions ${ }^{3-6}$. Among these compounds, $\mathrm{N}, \mathrm{N}^{\prime}$-dialkyl-N'-benzoylthioureas have been found to be useful ligands for the potential determination of traces of the transition metals by means of normal phase chromatography ${ }^{7}$. These reagents have also been shown to selectively extract several of the platinum group metals in the form of stable neutral chelates ${ }^{8}$ and their complexation capacity 9.10 .

Grigg et al have reported a range of thermal and metal catalysed imine -azomethine ylides-1,3-Dipolar cycloaddition cascades reactions furnishing azomethine ylides and their cycloadduct pyrrolidine compounds derived from amine and $\alpha$-aminoasit ester in good to excellent yield ${ }^{11-14}$. This cascade chemistry has provided a series of cephalosporin analogues 15 , spirobenzodiazepines related to MK-32913,14 and a potentially flexible entry into the complex molecular framework of natural products ${ }^{15-18}$

In a recent paper we reported a facile route conformationally constrained R-pro-S-pro peptidomimetics via thermal and metal catalysed imine -azomethine ylides-1,3-Dipolar Cycloaddition reaction in good to excellent yield ${ }^{17}$. We have also reported some novell nikkomycin analogous ${ }^{18}$ via cycloaddition of stabilised and nonstabilised azomethine ylides derived from Uracil Polyyoxin C.

\section{Results and Discussion:}

In view of these favourable properties of these ligands, we now report the reaction of benzoylisothiocyanate with substitueted pyrrolidines derived from $\alpha$-aminoasit ester via imine -azomethine 
ylides-1,3-Dipolar Cycloaddition. The highly functionalized benzoylaminocarbothioyl pyrrolidines were obtained under mild conditions in good to excellent yield.

$\mathrm{HCl}$<smiles>[R]C(N)C(C)=O</smiles>

(1)
$\mathrm{Ph}-\mathrm{CHO}$

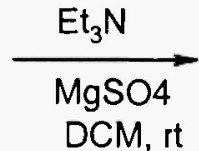

$\mathrm{Ph}$<smiles>[R]C(N=Cc1ccccc1)C(C)=O</smiles>

(2)

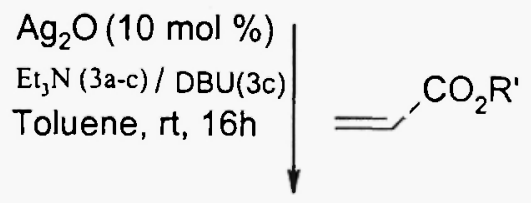<smiles>[R]OC(=O)[C@H]1CC([R])(C(C)=O)N(C(=S)NC([R])=O)[C@H]1c1ccccc1</smiles>

(4)a. $R=H, R^{\prime}=M e, R^{\prime \prime}=P h \quad 98 \%$

b. $\mathrm{R}=\mathrm{Ph}, \mathrm{R}^{\prime}=\mathrm{Me}, \mathrm{R}^{\prime \prime}=\mathrm{Ph} 95 \%$

c. $\mathrm{R}=\mathrm{CH}_{2} \mathrm{Ph}, \mathrm{R}^{\prime}=\mathrm{Me}, \mathrm{R}^{\prime \prime}=\mathrm{Ph} 94 \%$

d. $R=$ Me, R' = Menthyl, $R^{\prime \prime}=P h \quad 90 \%$<smiles>[R]OC(C)(O)[C@H]1C[C@H](C([R])=O)[C@H](c2ccccc2)N1</smiles>

(3) a. $R=H, R^{\prime}=\mathrm{Me} \quad 85 \%$

b. $R=P h, R^{\prime}=M e \quad 94 \%$

c. $\mathrm{R}=\mathrm{CH}_{2} \mathrm{Ph}, \mathrm{R}^{\prime}=\mathrm{Me} 90 \%$

d. $R=M e, R^{\prime}=$ Menthy! $92 \%$<smiles>C=CC(=O)O[C@H]1C[C@H](C)CCC1C(C)C</smiles>

(5) (1R, 2S, 5R-(-)-menthyl acrylate

\section{Scheme 1}

Thus the substituted pyrrolidines (3a-c) were prepared from the corresponding imines $(2 \mathbf{a}-\mathbf{c})^{12.19}$ in 85 $94 \%$ yield as autlined in scheme 1. The reaction of these pyrolidine cycloadducts (3a-c) with benzoylisothiocyanate in acetonitrile at ambient temperature for 3-4 h gave benzoylaminocarbothioyl pyrrolidine (4a-c) in $90-98 \%$ yield (Scheme 1). An example of chiral version of benzoylaminocarbothioyl pyrrolidine derivative was also reported. This was prepared from chiral pyrrolidine (3d) oryginated from menthyl acrylate as dipolarophile via cycloaddition cascade pocesses. ${ }^{12.19}$ The cycloadduct was then treated with benzoylisothiocyanate under similar conditions ( $\mathrm{MeCN}, \mathrm{rt}, 4 \mathrm{~h})$ to afford chiral benzoylaminocarbothioyl pyrrolidinc (4d) in $92 \%$ yield as a 3:1 rotomer (scheme 1). The structure and rotomer ratio of (4a-d) were detemined on the basis of ${ }^{1} \mathrm{Hnmr},{ }^{13} \mathrm{Cnmr}$, DEPT and ${ }^{2} \mathrm{D}$-COSY studies (see experimental). The stereochemistry of substitution on pyrrolidine ring was assigned from n.O.e. data and by the related previous 
work $^{14-18}$ and confirmed as reported before ${ }^{12-17}$. Note that (4d) depicts the absolute stereochemistry of cycloadducts derived from $1 \mathrm{R}, 2 \mathrm{~S}, 5 \mathrm{R}-(-)$-menthyl acrylate (5) (see experimental).

In summary, the versatility of technically simple metal catalysed imine -azomethine ylides-1,3-Dipolar cycloaddition cascades applied to prepare some novel highly functionalized and potential bioactive chiral and achiral benzoylaminocarbothioyl pyrrolidines. The compound were obtained under mild conditions in excellent yield and provides an major increase in molecular complexity. Some more chiral version of these processes and their metal complexes which can be used as chiral catalyst are under investigation. The bioactivities of the final compounds will be done in conjuntion with a pharmaceutical company.

Acknowledgement: We thank The Scientific and Technical Research Council of Turkey (TUBITAK) (Project no: TBAG-2154, 102T033) and Mersin University for support.

\section{Experimental}

General Technical Data: IR spectra were recorded in the $4000-400 \mathrm{~cm}^{-1}$ range on a Shimadzu 435 spectrophotometer, using $\mathrm{KBr}$ pellets. Nuclear magnetic resonance spectra and decoupling experiments were determined at $300 \mathrm{MHz}$. on a Q.E 300 instrument and at $500 \mathrm{MHz}$ on a Bruker AM500 spectrometer as specified. Chemical shifts are given in parts per million $(\delta)$ downfield from tetramethylsilane as internal standard. Spectra were determined in deuteriochloroform except where otherwise stated. The following abbreviations are used; $s=$ singlet, $d=$ doublet, $\mathrm{t}=$ triplet, $\mathrm{q}=$ quartet, $\mathrm{m}=$ multiplet, $\mathrm{b}=$ broad and brs $=$ broad singlet. Flash column chromatography was performed using silica gel 60 (230-400 mesh). Kieselgel columns were packed with silica gel GF254 (Merck 7730). Petroleum ether refers the fraction with b.p $40-60{ }^{\circ} \mathrm{C}$ unless otherwise specified. Melting points were determined on a digital electrothermal model 9200 hot stage apparatus and are uncorrected. Microanalyses were obtained using a Carlo - Erba Model 1106 instrument. Mass spectra were recorded at $70 \mathrm{ev}$ on a VG Autospec mass spectrometer. Specific rotations were measured at ambient temperature with an Optical Activity Ltd., AA-1000 polarimeter. All solvents were purified according to procedures given in Purification of Laboratory Chemicals, D.D. Perrin, W.L.F. Armarego, D.R. Perrin, Permagon Press, 1980. The cycloadduct $(3 \mathrm{~d})^{19}$ was prepared from $1 \mathrm{R}, 2 \mathrm{~S}, 5 \mathrm{R}$-menthyl acrylate. The menthyl acrylate derived from $(1 \mathrm{R}, 2 \mathrm{~S}, 5 \mathrm{R})$-menthol which was readily synthesised from a reported method ${ }^{20}$ by transesterification of ethyl acrylate using a Grignard complex of menthol. Benzoylisothiocyanate was prepared accordig to litterature procedure ${ }^{2}$. 
General Procedure for the synthesis of Benzoylaminocarbothioyl pyrrolidines. To a sitirred solution of benzoylisothiocyanate $(1.22 \mathrm{mmol})$ in freshly distilled dry degassed acetonitrile $(20 \mathrm{ml})$ was added a solution of pyrrolidine cycloadduct $(1.22 \mathrm{mmol})$ in acetonitrile $(10 \mathrm{ml})$ during $15 \mathrm{~min}$. and stirred at ambient temperature under a nitrogen atmosphere for 3-5 h. The solvent was evaporated under reduced pressure (bath temperature not higher than $30^{\circ} \mathrm{C}$ ) and the residue was crystallised from an appropriate solvent.

1-Benzoylaminocarbothioyl-5-phenyl-pyrrolidine-2,4-dicarboxylic acid dimethyl ester (4a) After a reaction time of $2 \mathrm{~h}$ and work up the product as 4:1 mixture of rotomer crystallised from petroleum etherhexane as a pale yellow amorphous solid in $98 \%$ yield. The rotomeric mixture dissapear when heated to $323 \mathrm{~K}$ in $\mathrm{CDCl}_{3}$. HRMS (EI) found: 426.4865, $\mathrm{C}_{22} \mathrm{H}_{20} \mathrm{~N}_{2} \mathrm{O}_{5} \mathrm{~S}$ requires: 426.4864. m/z (\%) (ES): $427(\mathrm{M}+1,100)$, 264(4) and 143(3). $V_{\max }(\mathrm{KBr}): 3300,2929,1690$ and $1074 \mathrm{~cm}^{-1} . \delta_{\mathrm{H}}(500 \mathrm{MHz})\left(\mathrm{CDCl}_{3}\right)(323 \mathrm{~K}): 8.17$ (brs, 1H, NH), 7.62-7.19(m, 10H, ArH), 5.60 (brd, 1H, J $7.5 \mathrm{~Hz}, 5-\mathrm{H}$ ), 4.90 (brd, 1H, J 8.2 Hz, 2-H), 3.82(s, 3H, $\mathrm{OMe}), 3.62(\mathrm{~m}, 1 \mathrm{H}, 4-\mathrm{H}), 3.33(\mathrm{~s}, 3 \mathrm{H}, \mathrm{OMe}), 2.70(\mathrm{dd}, 1 \mathrm{H}, \mathrm{J} 6.1$ and $13.2 \mathrm{~Hz}, 3 \mathrm{~b}-\mathrm{H}), 2.44(\mathrm{dd}, 1 \mathrm{H}, \mathrm{J} 7.2$ and $13.6 \mathrm{~Hz}, 3 \mathrm{a}-\mathrm{H}) . . \delta_{\mathrm{C}}\left(\mathrm{CDCl}_{3}\right)(323 \mathrm{~K}): 178.8(\mathrm{C}=\mathrm{S}), 170.7(\mathrm{C}=\mathrm{O}), 169.14(\mathrm{C}=\mathrm{O}), 164.09(\mathrm{C}=\mathrm{O}), 136.4,133.1$, $132.76,129.1,128.9,128.7(2 \mathrm{C}), 127.8(2 \mathrm{C}), 127.4,68.164 .5,62.9,52.5,51.4,48.9,48.2,29.8$. N.O.e data: Irradiation of $5-\mathrm{H}$ effected enhancement of $3 \mathrm{a}-\mathrm{H}(6)$ and $4-\mathrm{H}(8)$ and $2-\mathrm{H}(4)$, Irradiation of $4-\mathrm{H}$ effected enhancement of $3 \mathrm{a}-\mathrm{H}(7)$ and $5-\mathrm{H}(9)$.

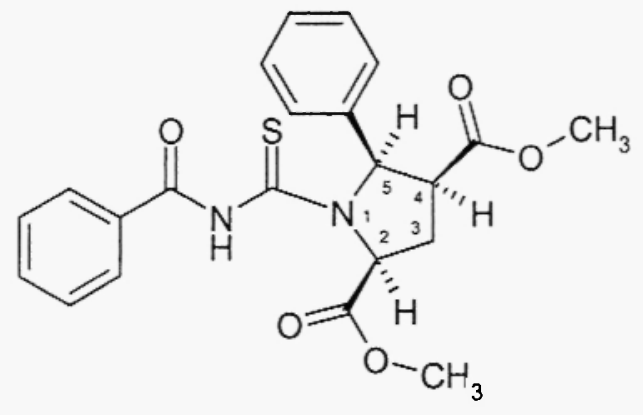

I-Benzoylaminocarbothioyl-2,5-diphenyl-pyrrolidine-2,4-dicarboxylic acid dimethyl ester (4b). After a reaction time of $2.5 \mathrm{~h}$ and work up the product as 3:1 mixture of rotomer crystallised from petroleum etherhexane as a pale yellow amorphous solid in $95 \%$ yield. HRMS (EI), found: $502.2827, \mathrm{C}_{28} \mathrm{H}_{26} \mathrm{~N}_{2} \mathrm{O}_{5} \mathrm{~S}$ requires: 502. $2825 ; \mathrm{m} / \mathrm{z}(\%)(\mathrm{ES}): 503(\mathrm{M}+1,100)$ and 143(60). $v \max (\mathrm{KBr}): 3306,2930$ and $1696 \mathrm{~cm}^{-1} . \delta_{\mathrm{H}}(500$ $\mathrm{MHz})\left(\mathrm{CDCl}_{3}\right):$ 7.86(brs, $\left.1 \mathrm{H}, \mathrm{NH}\right), 7.78(\mathrm{~m}, 2 \mathrm{H}, \mathrm{Ar}-\mathrm{H}), 7.70(\mathrm{~m}, 2 \mathrm{H}, \mathrm{Ar}-\mathrm{H}), 7.60(\mathrm{~m} .2 \mathrm{H}, \mathrm{Ar}-\mathrm{H}), 7.52-7.15(\mathrm{~m}$, $9 \mathrm{H}, \mathrm{ArH}), 5.85(\mathrm{~d}, 1 \mathrm{H}, \mathrm{J} 8.8 \mathrm{~Hz}, 5-\mathrm{H}$, minor rotomer), $5.80 .(\mathrm{d}, 1 \mathrm{H}, \mathrm{J} 9.0 \mathrm{~Hz}, 5-\mathrm{H}$, major rotomer), $3.90(\mathrm{~s}, 3 \mathrm{H}$, OMe major rotomer), 3.69(s, $3 \mathrm{H}$, OMe minor rotomer), $3.42(\mathrm{~m}, 1 \mathrm{H}, 4-\mathrm{H}), 3.38(\mathrm{~m}, 1 \mathrm{H} .3 \mathrm{~b}-\mathrm{H}) .3 .31(\mathrm{~s}, 3 \mathrm{H}$, OMe minor rotomer), 3.22(s, $3 \mathrm{H}$, OMe major rotomer), $2.55(\mathrm{~m}, 1 \mathrm{H}, 3 \mathrm{a}-\mathrm{H}) . \delta_{\mathrm{C}}\left(\mathrm{CDCl}_{3}\right): 187.0$ and $179.7(\mathrm{C}=\mathrm{S}$, rotomer), 174.8 and $172.1(\mathrm{C}=\mathrm{O}$, rotomer), 170.2 and $169.4(\mathrm{C}=\mathrm{O}$, rotomer $), 168.7$ and $164.6(\mathrm{C}=\mathrm{O}$, rotomer $)$. 
<smiles>COC(=O)[C@H]1C[C@@H](c2ccccc2)[C@](C(=O)OC)(c2ccccc2)N1C(=S)NC(=O)c1ccccc1</smiles>

1-Benzoylaminocarbothioyl-2-benzyl-5-phenyl-pyrrolidine-2,4-dicarboxylic acid dimethyl ester (4c). After a reaction time of $4 \mathrm{~h}$ and work up the product as 2:1 mixture of rotomer crystallised from petroleum ether-hexane as a pale yellow amorphous solid in $94 \%$ yield.. HRMS (EI), found: $516.6093 . \mathrm{C}_{29} \mathrm{H}_{28} \mathrm{~N}_{2} \mathrm{O}_{5} \mathrm{~S}$ requires: 516.6090. $\mathrm{m} / \mathrm{z}(\%)(\mathrm{ES}): 517(\mathrm{M}+1,100), 319(5)$ and 143(6). $\quad V \max (\mathrm{KBr}): 3290,2890$ and 1702 $\mathrm{cm}^{-1} . \delta_{\mathrm{H}}(500 \mathrm{MHz})\left(\mathrm{CDCl}_{3}\right): 8.15(\mathrm{~d}, 1 \mathrm{H}, \mathrm{Ar}-\mathrm{H}), 7.93(\mathrm{brs}, 1 \mathrm{H}, \mathrm{NH}), 7.51-7.01(\mathrm{~m}, 14 \mathrm{H}, \mathrm{ArH}), 5.25 .(\mathrm{d}, 1 \mathrm{H}, \mathrm{J}$ $9.95 \mathrm{~Hz}, 5-\mathrm{H}$, minor rotomer), $5.17 \mathrm{~d}, 1 \mathrm{H}, \mathrm{J} 10.0 \mathrm{~Hz}, 5-\mathrm{H}$, major rotomer), 4.63(d, 1H, J12.4 Hz, 4-H major rotomer), 4.23(d, $1 \mathrm{H}, \mathrm{J} 12.6 \mathrm{~Hz}, 4-\mathrm{H}$ minor rotomer), 3.93(s, 3H, OMe major rotomer), 3.79(s, 3H, OMe minor rotomer), 3.45 and $2.95\left(2 \times d, 2 \times 1 \mathrm{H}, \mathrm{J} 13.3 \mathrm{~Hz}, \mathrm{CH}_{2} \mathrm{Ph}\right.$ minor rotomer), 3.33 and $2.85(2 \times d, 2 \times 1 \mathrm{H}, \mathrm{J} 13.5 \mathrm{~Hz}$, $\underline{\mathrm{CH}}_{2} \mathrm{Ph}$ major rotomer), 3.11 (s, 3H, OMe minor rotomer), 3.05(s, 3H, OMe major rotomer), 2.52-2.15(m, 2H, $3 \mathrm{~b}-\mathrm{H}$ and $3 \mathrm{a}-\mathrm{H}), \delta_{\mathrm{C}}\left(\mathrm{CDCl}_{3}\right): 186.5$ and $177.9(\mathrm{C}=\mathrm{S}$, rotomer $), 173.4$ and $172.0(\mathrm{C}=\mathrm{O}$, rotomer $), 169.7$ and 169.2( $\mathrm{C}=\mathrm{O}$, rotomer), 163.9 and $163.4(\mathrm{C}=\mathrm{O}$, rotomer). N.O.e data: Irradiation of $4-\mathrm{H}$ effected enhancement of $3 \mathrm{a}-\mathrm{H}(5)$ and $5-\mathrm{H}(6)$.<smiles>COC(=O)[C@H]1C[C@@](Cc2ccccc2)(C(=O)OC)N(C(=S)NC(=O)c2ccccc2)[C@H]1c1ccccc1</smiles>

(2S,4S,5R)-1-Benzoylaminocarbothioyl-2-methyl-5-phenyl-pyrrolidine-2,4-dicarboxylic acid 4((IR,2S,5R)-2-isopropyl-5-methyl-cyclohexyl) ester 2-methyl ester (4d). After a reaction time of 4 h and work up the product as 3:1 mixture of rotomer crystallised from petroleum ether-hexane as a pale yellow amorphous solid in $90 \%$ yield. $[\alpha]_{D}{ }^{20}-22.3(0.5 \mathrm{~g} / 100 \mathrm{ml}$, EtOH). HRMS (EI), found: 565. 7442, $\mathrm{C}_{32} \mathrm{H}_{41} \mathrm{~N}_{2} \mathrm{O}_{5} \mathrm{~S}$ requires: $565.7444 . \mathrm{m} / 2(\%)(\mathrm{ES}): 565(\mathrm{M}+1,100)$ and $402(13) . v \max (\mathrm{KBr}): 3306,2929$ and 
$1074 \mathrm{~cm}^{-1} . \delta_{\mathrm{H}}(500 \mathrm{MHz})\left(\mathrm{CDCl}_{3}\right): 8.10(\mathrm{~m}, 1 \mathrm{H}, \mathrm{Ar}-\mathrm{H}), 7.95(\mathrm{brs}, 1 \mathrm{H}, \mathrm{NH}), 7.65-7.25(\mathrm{~m}, 9 \mathrm{H}, \mathrm{ArH})$, 5.82.(d, $1 \mathrm{H}, \mathrm{J} 9.4 \mathrm{~Hz}, 5-\mathrm{H}$, minor rotomer), 5.60.(d, $1 \mathrm{H}, \mathrm{J} 9.5 \mathrm{~Hz}, 5-\mathrm{H}$, major rotomer), $5.60(\mathrm{~m}, 1 \mathrm{H}, \mathrm{OCH}$ menthyl), $3.87(\mathrm{~s}, 3 \mathrm{H}, \mathrm{OMe}$ major rotomer), 3.80(m, $1 \mathrm{H}, 4-\mathrm{H}), 3.74(\mathrm{~s}, 3 \mathrm{H}, \mathrm{OMe}$ minor rotomer $), 3.05(\mathrm{~m}, 1 \mathrm{H}, 3 \mathrm{~b}-\mathrm{H})$, 2.22(m, 1H, 3a-H), 1.94(s, 3H, Me, major rotomer), $1.90(\mathrm{~s}, 3 \mathrm{H}, \mathrm{Me}$, minor rotomer), $1.50(\mathrm{~m}, 3 \mathrm{H}$, menthly-H), $1.18(\mathrm{~m}, 2 \mathrm{H}$, menthyl- $\mathrm{H}), 0.95(\mathrm{~m}, 1 \mathrm{H}$, menthyl $-\mathrm{H}), 0.90-0.75(\mathrm{~m}, 3 \mathrm{H}$, menthyl-H), 0.82 and $0.73(2 \mathrm{xd}, 2 \times 3 \mathrm{H}, \mathrm{J}$ $6.4 \mathrm{~Hz}, \mathrm{CHMe}_{2} .0 .56(\mathrm{~d}, 3 \mathrm{H}, \mathrm{J} 7.0 \mathrm{~Hz}, \mathrm{Me}) . \delta_{\mathrm{C}}\left(\mathrm{CDCl}_{3}\right): 185.3$ and $177.7(\mathrm{C}=\mathrm{S}$, rotomer $), 173.4$ and $172.3(\mathrm{C}=\mathrm{O}$, rotomer), 171.1 and $168.4(\mathrm{C}=\mathrm{O}$, rotomer), 167.2 and $164.4(\mathrm{C}=\mathrm{O}$, rotomer $)$ N.O.e data: Irradiation of $2-\mathrm{Me}$ effected enhancement of $3 \mathrm{a}-\mathrm{H}(8)$ and $4-\mathrm{H}(4)$ and $5-\mathrm{H}(2)$, Irradiation of 4-H effected enhancement of 3a-H(5) and nd $5-\mathrm{H}(6)$.<smiles>COC(=O)[C@]1(C)C[C@@H](C(=O)O[C@H]2C[C@H](C)CCC2C(C)C)[C@H](c2ccccc2)N1C(=S)NC(=O)c1ccccc1</smiles>

\section{REFERENCES:}

1. (a) Gutt Guttmann, M.; Lubert, K.H.; Beyer, L. Fresenius J Anal. Chem. 1996, 356, 263 (b) Shen, X.; Shi, X.; Kang, B.; Tong, Y.; Liu, Y.; Gu, L.; Liu, Q.; Huang, Y. Polyhedron 1998, 18, 33 (c) Brindley, J.C.; Caldwell, J.M.; Meakins, G.D.; Plackett, S.J.; Price, J.P. J. Chem. Perkin Trans I, $1987,1153$.

2. (a) Arslan, H.; Florke, U.; Kulcu, N. Transit. Metal Chem. 2003, 28, 816. (b) Mohamadou, A.; Dechamps-Olivier, I.; Barbier, J.P. Polyhedron 1994, 13, 3277. (c) Dechamps-Olivier, 1.; Guillion, E.; Mohamadou, A.; Barbier, J.P. Polyhedron 1996, 15, 3617.

3. (a) Frech, F.A.; Blanz, E.J.; Cancer Res. 1965, 25, 1454. (b) Frech, F.A.; Blanz, E.J.; J. Med.Chem. 1966, 9, 585 .

4. (a) Blanz, E.J.; Frech, F.A.; Cancer Res. 1968, 28, 2419. (b) Frech, F.A.; Blanz, E.J.; Amaral, J.R.D.; French, D.A. J. Med.Chem. 1970, 13, 1117.

5. Sacht, C.; Datt, M M.S.; Otto, S.; Roodt, A.; J. Chem. Soc. Dalton Trans, 2000, 727.

6. Nicolae, R.; Lucica, B.; Revista de Chimie.; 2002, 53(11), 758.

7. König, K.H.; Schuster, M.; Schneeweis, G.; Steinbrech, B. Fresenius'Zeit. Anal. Chem.. 1984. 319, 66.

8. König, K.H.; Schuster, M.; Schneeweis, G.; Steinbrech, B. Schlodder, R.; Fresenius' Zeit. Anal. Chem. 1985, 321, 457. 
9. Schuster, M.; Kugler, B.; König, K.H.; J. Anal. Chem.. 1990, 338, 717.

10. Schuster, M.; J. Anal. Chem.. 1992, 342, 791.

11. Grigg, R.; Sridharan, V.; Adv. in Cycloaddition,, JAI Press Inc., 1993, Vol.3, 161-204

12. Barr, D. A.; Dorrity, M.J., Grigg, R.; Hargreaves, S.; Malone, J. F.; Montgomery, J.; Redpath, J.; Stevenson, P.; Thomton-Pett, M.; Tetrahedron, 1995, 51, 273. Barr, D. A.; Dorrity, M.J., Grigg, R.; Gunaratne, H.Q.N.; Kemp, J.; McMeekin, P Sridharan, V Tetrahedron, 1988, 44, 557. Döndaş, H.A. Grigg, R. Maclachton W.S. Macperson D.T.; Markandu, J.; Sridharan,V.; Suganthan, S.; Tetrahedron Letters, 2000, 41, 967. Dondas, H.A.; Grigg, R.; Thomton-Pett; M.; Tetrahedron, 1996, 52,13455 .

13. Dondas, H.A.; Sonmez, S.; Heterocylic Comminications, 2003, 9, 23.

14. Dondas, H.A., Duraisingham, J.; Grigg, R.; MacLaclan W.S. MacPherson D.T. ; Thornton-Pett, M. Sridharan,V.; Suganthan, S. Tetrahedron, 2000, 56, 4063-407 Grigg, R.;McMeekin, P.; Sridharan, V.; Tetrahedron, 1995, 51, 13347-13356

15. Cooper, D.M.; Grigg, R.; Hargreaves, S.; Kennewell, P.; Redpath, J; Tetrahedron. 1995, 51, 7791. Grigg, R. Thornton - Pett, M.;Xu, J;Xu, L-H Tetrahedron, 1999,.55, 13841

16. Dondas, H.A.; Grigg, R.; Killner, C.; Tetrahedron, 2003, 59, 8481-8487

17. Dondas, H.A.; Grigg, R.; Killner, C.; Tetrahedron, 2004, in press.

18. Dondas, H.A.; Grigg, R.; unpublished results.

19. Frank, R.; Davis, H. Drake, S.; McPherson, J.; J. Am. Chem. Soc., 1944, 66. 1509.

\section{Received on November 2, 2003.}


